Research article

\title{
Generic and disease-specific health related quality of life in non-cirrhotic, cirrhotic and transplanted liver patients: a cross-sectional study
} Simone $M$ van der Plas ${ }^{1}$, Bettina E Hansen*2, Josien B de Boer ${ }^{3}$, Theo Stijnen ${ }^{4}$, Jan Passchier ${ }^{5}$, Robert A de Man ${ }^{6}$ and Solko W Schalm ${ }^{7}$

\begin{abstract}
Address: ${ }^{1}$ Department of Gastroenterology and Hepatology, Erasmus Medical Centre Rotterdam, P.O box 2040, 3000 CA Rotterdam, The Netherlands, ${ }^{2}$ Department of Epidemiology and Biostatistics, Erasmus Medical Centre Rotterdam, P.O. box 1738, 3000 DR Rotterdam, The Netherlands, ${ }^{3}$ TNO Prevention and Health, Division Child Health, Gorter Building, P.O. box 2215, 2301 CE Leiden, The Netherlands, ${ }^{4}$ Department of Epidemiology and Biostatistics, Erasmus Medical Centre Rotterdam, P.O. box 1738, 3000 DR Rotterdam, The Netherlands, ${ }_{5}^{5}$ Department of Medical Psychology and Psychotherapy, Erasmus Medical Centre Rotterdam, P.O. box 1738, 3000 CA Rotterdam, The Netherlands, ${ }^{6}$ Department of Gastroenterology and Hepatology, Erasmus Medical Centre Rotterdam, P.O box 2040, 3000 CA Rotterdam, The Netherlands and 7Department of Gastroenterology and Hepatology, Erasmus Medical Centre Rotterdam, P.O box 2040, 3000 CA Rotterdam, The Netherlands

Email: Simone M van der Plas - s.vanderplas@erasmusmc.nl; Bettina E Hansen* - b.hansen@erasmusmc.nl; Josien B de Boer - JB.deBoer@pg.tno.nl; Theo Stijnen - t.stijnen@erasmusmc.nl; Jan Passchier - j.passchier@erasmusmc.nl; Robert A de Man -r.deman@erasmusmc.nl; Solko W Schalm - s.schalm@erasmusmc.nl

* Corresponding author
\end{abstract}

Published: 17 November 2003

BMC Gastroenterology 2003, 3:33
Received: 23 July 2003

Accepted: 17 November 2003

This article is available from: http://www.biomedcentral.com/I47I-230X/3/33

(C) 2003 van der Plas et al; licensee BioMed Central Ltd. This is an Open Access article: verbatim copying and redistribution of this article are permitted in all media for any purpose, provided this notice is preserved along with the article's original URL.

\begin{abstract}
Background: Studies on Health Related Quality of Life (HRQoL) of chronic liver patients were performed in clinical populations. These studies included various disease stages but small variations in aetiology and no transplanted patients. We performed a large HRQoL study in non-cirrhotic, cirrhotic and transplanted liver patients with sufficient variety in aetiology. We compared the generic HRQoL and fatigue between liver patients and healthy controls and compared the diseasespecific and generic HRQoL and fatigue between non-cirrhotic, cirrhotic and transplanted liver patients, corrected for aetiology.
\end{abstract}

Methods: Members of the Dutch liver patient association received the Short Form-36, the Liver Disease Symptom Index and the Multidimensional Fatigue Index-20. Based on reported clinical characteristics we classified respondents $(n=1175)$ as non-cirrhotic, compensated cirrhotic, decompensated cirrhotic or transplants. We used linear, ordinal and logistic regression to compare the HRQoL between groups.

Results: All liver patients showed a significantly worse generic HRQoL and fatigue than healthy controls. Decompensated cirrhotic patients showed a significantly worse disease-specific and generic HRQoL and fatigue than non-cirrhotic patients, while HRQoL differences between noncirrhotic and compensated cirrhotic patients were predominantly insignificant. Transplanted patients showed a better generic HRQoL, less fatigue and lower probabilities of severe symptoms than non-cirrhotic patients, but almost equal probabilities of symptom hindrance.

Conclusions: $\mathrm{HRQ} \mathrm{LL}$ in chronic liver patients depends on disease stage and transplant history. Non-cirrhotic and compensated cirrhotic patients have a similar HRQoL. Decompensated patients 
show the worst HRQoL, while transplanted patients show a significantly better HRQoL than cirrhotic and non-cirrhotic patients.

\section{Background}

In the year $2000,40.9 \%$ of the Dutch population suffered from a chronic disease. In that same year, more than 800 Dutch men and women died of a chronic liver disease ( $0.6 \%$ of year specific total mortality) [1]. Until today, the Dutch liver patient association (Nederlandse Leverpatiënten Vereniging (NLV)) and many other patient associations fight for recognition of disease related physical, mental and social problems of chronic patients. Quality of life research could contribute to a better understanding of these problems and may fulfil this quest for recognition.

One of the first studies done on Health Related Quality of Life (HRQoL) of chronic liver patients was conducted in 1979 and studied the effect of liver transplantation on HRQoL of chronic liver patients [2]. The study demonstrated that the quality of life of liver patients after transplantation ranged from poor to superior. In 1998, Foster et al compared the HRQoL of liver patients with viral hepatitis $\mathrm{B}$ and $\mathrm{C}$ and reported that social functioning, energy and fatigue and role limitations due to physical problems were significantly more impaired in hepatitis $C$ patients [3]. In more recent studies, the HRQoL of different stages of liver disease were compared. Younossi et al found an increasing impairment of generic HRQoL with increasing disease severity, while Marchesini et al found that the most relevant determinants of impaired health status were severity of disease and muscle cramps [4-6].

These studies contributed substantially to our knowledge of the physical, social and mental problems of chronic liver patients. However, the majority of these studies was conducted in relatively small clinical populations and the comparisons between disease stages were adjusted for small or few aetiological groups. Moreover, none of these studies included liver transplant recipients in the study population.

Therefore, to get a better understanding of the differences in HRQoL between the various disease stages and the relation with transplanted liver patients, one must study a large liver patient population with a broad variety with respect to disease stage and aetiology. Furthermore, the HRQoL should be measured by a generic as well as a disease-specific questionnaire to give a profound insight in the differences in HRQoL between disease stages $[7,8]$.

Our study offers an extensive overview of the HRQoL of chronic liver patients. In contrast to the clinical popula- tions in earlier studies, our collaboration with the Dutch liver patient association (NLV) gave us the opportunity to study the HRQoL of large number liver patients, approaching a population level. The study population of 1175 members included various stages of cirrhosis and aetiologies as well as a large number of transplanted liver patients. It provided us with sufficient varied information to realise an HRQoL comparison between non-cirrhotic, compensated cirrhotic, decompensated cirrhotic and transplanted liver patients, corrected for aetiology.

Another distinguishing feature of this study is that the HRQoL information was generated by means of the Liver Disease Symptom Index 2.0 (LDSI), the Short Form-36 (SF-36) and the Multidimensional Fatigue Index-20 (MFI20). Other studies already used a combination of a generic and a disease-specific questionnaire $[4,5]$. However, the LDSI provides, in contrast to other liver disease-specific questionnaires, information about the severity of symptoms and hindrance of these symptoms during daily activities. In an earlier study we demonstrated that the LDSI provides additional information on top of the SF-36 and the MFI-20 (S.M. van der Plas, unpublished). The SF-36 and MFI-20 were both validated in a clinical chronic liver patient population [9]. Therefore, the combination of these instruments forms a reliable and valid method to accomplish the following aims:

1) To compare the generic HRQoL and fatigue between chronic liver patients and healthy Dutch controls and 2) to evaluate the differences in disease-specific HRQoL, generic HRQoL and fatigue between non-cirrhotic, compensated cirrhotic, decompensated cirrhotic and transplanted liver patients, corrected for aetiology. By evaluating the differences in disease-specific HRQOL, we addressed the known groups validity of the LDSI across the various subgroups. The known groups validity is based on the principle that certain specified groups of patients, may be anticipated to score differently from others. We evaluated whether the LDSI was sensitive to these differences.

\section{Methods \\ Study population}

In October 2000, all 2020 members of the NLV were approached for participation in this study and received a questionnaire by mail. The members included patients with a (history of) liver disease as well as non-patients who joined the NLV because of involvement with liver patients in family, circle of acquaintances or work. After 
two months, non-responders received a new questionnaire. We closed the response period 5 months after the first mailing. As requested by the Ethics Committee, members gave their informed consent by confirming their willingness to participate in the first question of the questionnaire.

Inclusion criteria were: 1) Informed consent, 2) having a (history of) liver disease and 3) aged 18 years or older at the moment of administration. To preserve the anonymity of the participants, the NLV withheld the coding of respondent numbers and member names, while the researcher withheld the completed questionnaires. The protocol was conform the ethical guidelines of the 1996 Declaration of Helsinki and has been approved by the Ethics Committee of the Erasmus Medical Centre Rotterdam, the Netherlands.

\section{Measurement Instruments}

The disease-specific LDSI 2.0 includes 18 items. Nine items measure the severity of: 'Itch', 'Joint pain', 'Pain in the right upper abdomen', 'Sleepiness during the day', 'Worry about family situation', 'Decreased appetite', 'Depression', 'Fear of complications' and 'Jaundice'. Nine other items measure the hindrance of these symptoms during daily activities. All items have 'the last week' as time frame and are scored on a 5-point scale ranging from 'not at all' to 'to a high extent'.

Apart from the LDSI, 6 additional items recommended by the NLV, were scored on the same 5-point scale. These items concerned: 'Memory problems due to liver disease', 'Change of personality due to liver disease', 'Hindrance in financial affairs due to liver disease', 'Involuntary change in use of time personality due to liver disease', 'Decreased sexual interest' and 'Decreased sexual activity'. The LDSI as well as the extra NLV items have recently been validated in chronic liver patients and showed a good feasibility, testretest reliability and construct validity (Van der Plas, unpublished).

The generic (Dutch) SF-36 version 1.2, includes 8 multiitem scales on Physical Functioning, Role limitations due to Physical problems, Bodily Pain, General Health, Vitality, Social Functioning, Role limitations due to Emotional problems and Mental Health. The scale scores range from 0 to 100 . A higher score indicates a better generic HRQoL. SF-36 data of Dutch healthy controls was available [10].

The domain-specific MFI-20 includes five 4-item scales: General Fatigue, Physical Fatigue, Reduction in Activity, Reduction in Motivation and Mental Fatigue and scale scores range from 4 to 20 . Higher scores indicate more fatigue. MFI-20 data of Dutch healthy controls was avail- able [11]. Both the SF-36 and the MFI-20 proved to be reliable and valid in Dutch chronic liver patients [9].

A separate questionnaire was used to determine sex, age, marital status, education level, aetiology, duration of the liver disease, status of the liver disease(s) (cured, noncured), presence of a liver transplant, presence of cirrhosis, presence or history of splenomegaly, ascites or oesophageal variceal bleedings, presence of oesophageal variceal bleedings or ascites in the year 2000, history of complications of cirrhosis (liver cancer or imminent coma), comorbidity (defined as the presence of diseases or disorders other than the liver disease that limit the respondent's daily functioning), medication use and the amount of hours per week spent on work and activities with and without physical effort.

\section{Liver patient comparison groups}

Due to the design of the study, respondents originated from all over the country and participated anonymously. Therefore, we based the categorisation of respondents in disease stage groups (non-cirrhotic (NC), compensated cirrhotic (CC), decompensated cirrhotic (DC)) or the liver transplant group (LTX) on reported clinical characteristics (table 1)

Furthermore, we categorised respondents in 5 aetiology groups based on reported aetiologies: Viral Hepatitis, Autoimmune Hepatitis, Cholestatic diseases, Hemochromatosis and other liver diseases. Transplanted respondents and respondents who considered themselves as cured were respectively assigned to the groups 'Liver transplants' and 'Cured liver diseases'.

We have validated the reliability of reported clinical characteristics, disease stage definitions and reported aetiologies in a pilot study conducted at our Hepatology outpatient clinic. Reported clinical characteristics and aetiologies demonstrated a good agreement between the test and the retest questionnaire (clinical characteristics: $\kappa$ $0.85[0.71,0.94]$ to $0.97[0.91,1.03]$; aetiologies: $\kappa 0.71$ $[0.63,0.79])$ and a good agreement with hospital data (clinical characteristics: $\kappa 0.68[0.45,0.90]$ to 0.71 [0.53, $0.88]$; aetiologies: $\kappa 0.63[0.55,0.78])$. Reported presence of cirrhosis showed a moderate agreement with hospital data ( $\kappa 0.52[0.31,0.73])$. The assigned disease stage groups showed a fair agreement with the disease stages based on hospital data of the patients ( $\kappa \quad 0.37$ [0.19, $0.55])$.

The hospital data revealed that our disease stage definitions (which during the pilot did not include the criterion of recent ascites or variceal bleeding), disregarded the temporary state of the decompensated cirrhotic stage: patients may become decompensated due to flare up of disease 
Table I: Classification of disease stage groups and the transplanted group based on reported clinical characteristics.

\begin{tabular}{lllllll}
\hline & $\begin{array}{l}\text { Reported } \\
\text { cirrhosis }\end{array}$ & $\begin{array}{l}\text { Reported } \\
\text { splenomegaly }\end{array}$ & $\begin{array}{l}\text { Reported } \\
\text { ascites }\end{array}$ & $\begin{array}{l}\text { Reported variceal } \\
\text { bleeding }\end{array}$ & $\begin{array}{l}\text { Reported ascites and/or } \\
\text { variceal bleeding in the } \\
\text { year 2000 }\end{array}$ \\
\hline HC & No & No & No & No & No & Nory \\
CC & Yes & - & - & - & No & No \\
CC & - & Yes & - & - & No & No \\
CC & - & - & Yes & - & No & No \\
CC & - & - & - & Yes & Yes & No \\
DC & - & - & - & - & - & Yes \\
LTX & - & - & - & - & & \\
\hline
\end{tabular}

NC $=$ Non-Cirrhosis, $C C=$ Compensated Cirrhosis, $D C=$ Decompensated Cirrhosis, $L T X=$ Liver transplanted. Legend clinical characteristics: No: Absence of the clinical characteristic is an absolute condition for the concerning disease stage group or transplant group. Yes: Presence of the clinical characteristic is an absolute condition for the concerning disease stage group or transplant group. - : Presence or absence of clinical characteristic is no absolute condition.

activity or inflammation, but can reverse to an apparently compensated state after treatment with beta-blockers, diuretics or (surgical) interventions.

During the current study, we took this temporary state of decompensated cirrhosis into account by including the criterion concerning: The presence of ascites or oesophageal variceal bleedings in the year 2000 (the year of the study), as extra item into the background questionnaire. This extra criterion distinguished decompensated cirrhotic patients from reversed decompensated cirrhotic patients. In the NLV population 43 compensated cirrhotic were defined as reversed decompensated cirrhotic patients (based on the absence of ascites and/or variceal bleedings in the year 2000 and the use of diuretics and/or propanolol at the moment of our study). The HRQoL level of these patients fitted the HRQoL level of the compensated cirrhotic group and not the HRQoL level of decompensated patients. This indicated that these patients were correctly categorised as compensated cirrhotic patients.

\section{Controls}

Healthy Dutch controls for the SF-36 ( $\mathrm{n}=1715)$ originated from a nationwide, population based health status survey with the standard version of the SF-36, conducted by the Dutch Organisation for Applied Scientific Research (TNO). Controls were adult members of a random sample of Dutch households, drawn from the national telephone registry. This registry included a somewhat larger percentage of men and a smaller category in the age of 15-25 years than the adult population in the Netherlands. TNO corrected for this imbalance by stating in the introductory letter that any adult member of the household could complete the questionnaire. A random set of introductory letters requested that the questionnaire had to be completed by a member of the household between the ages of 15-25 years [10].
Healthy Dutch controls for the MFI-20 ( $\mathrm{n}=139)$ originated from a study on fatigue and radiotherapy in cancer patients. Controls were adults from a non-selective sample of households taken from the telephone directories. As women are more frequently at home, researchers of this study prevented overrepresentation of women by interviewing the next person to have a birthday within that household [11].

\section{Statistical Methods}

We compared the generic HRQoL of NC, CC, DC and LTX with the generic HRQoL of the general Dutch population. SF-36 scale scores were calculated by SF-36 scoring algorithms [12]. We estimated mean SF-36 scale scores by general linear regression, in which we used the SF-36 scales as dependent outcome. A variable, which included the disease stage groups, transplanted group and controls served as independent determinant. Means were corrected for sex, age, marital status and education level. Furthermore, we compared fatigue between NC, CC, DC and LTX and the general Dutch population. The MFI-20 scale scores were calculated by MFI-20 scoring algorithms [13]. We used general linear regression with the MFI-20 scales as outcome to estimate mean MFI-20 scale scores. Again, the variable that included the various subgroups served as independent determinant. Means were corrected for sex, age and education level.

To compare the generic HRQoL and fatigue between NC, CC, DC and LTX, we performed a linear regression in SPSS 10.0 and in SAS 8.0. SF-36 scales or MFI-20 scales served as dependent outcomes. Mean differences in SF-36 scale scores or mean differences in MFI-20 scale scores were calculated between NC (reference) and CC, DC and LTX. Of each scale, model-based standard errors in SPSS were compared with robust standard errors provided by PROC MIXED using the 'empirical'-option in SAS 8.0. Model- 
Table 2: Demographic and clinical characteristics of respondents.

\begin{tabular}{|c|c|c|c|c|c|c|}
\hline Characteristic & $\begin{array}{l}\text { NC } \\
(n=489)\end{array}$ & $\begin{array}{l}C C \\
(n=391)\end{array}$ & $\begin{array}{l}D C \\
(n=84)\end{array}$ & $\begin{array}{l}\text { LTX } \\
(n=186)\end{array}$ & $\begin{array}{l}\text { Dutch SF-36 } \\
\text { controls } \\
(n=|7| 5)\end{array}$ & $\begin{array}{l}\text { Dutch MFI-20 } \\
\text { controls } \\
(n=139)\end{array}$ \\
\hline Mean age $\pm S D, y r$ & $48 \pm 12$ & $49 \pm 14$ & $50 \pm 12$ & $49 \pm 13$ & $48 \pm 17$ & $46 \pm 16$ \\
\hline \multicolumn{7}{|l|}{ Gender } \\
\hline Men, n (\%) & $214(43.8)$ & $162(41.4)$ & $36(42.9)$ & $78(41.9)$ & $967(56.6)$ & $60(44.4)$ \\
\hline Women, n (\%) & $275(56.2)$ & $229(58.6)$ & $48(57.1)$ & $108(58.1)$ & $740(43.4)$ & $75(55.6)$ \\
\hline \multicolumn{7}{|l|}{ Education } \\
\hline None/elementary education & $33(6.8)$ & $39(10.0)$ & $15(18.1)$ & $18(9.7)$ & $212(12.6)$ & II (8.I) \\
\hline Lower secondary education & $178(36.5)$ & $157(40.2)$ & $27(32.5)$ & $74(40.0)$ & $569(33.8)$ & $90(66.7)$ \\
\hline Upper/post secondary education & $|4|(28.9)$ & $106(27.1)$ & $24(28.9)$ & $48(25.9)$ & $477(28.4)$ & $34(25.2)$ \\
\hline |st/2nd stage tertiary education & $136(27.9)$ & $89(22.8)$ & $17(20.5)$ & $45(24.3)$ & $424(25.2)$ & $0(0)$ \\
\hline \multicolumn{7}{|l|}{ Marital status } \\
\hline Married / Living together & $360(73.9)$ & $292(75.1)$ & $57(67.9)$ & $139(75.1)$ & $1278(74.8)$ & \\
\hline Single / Widow(er) / Divorced & $127(26.1)$ & $97(24.9)$ & $27(32.1)$ & $46(24.9)$ & $431(25.2)$ & \\
\hline \multicolumn{7}{|l|}{ Aetiology } \\
\hline Viral hepatitis & $169(36.3)$ & $77(20.9)$ & $23(30.3)$ & & & \\
\hline Autoimmune hepatitis & $51(10.9)$ & $77(20.9)$ & $11(14.5)$ & & & \\
\hline $\mathrm{PBC} / \mathrm{PSC}$ & $76(16.3)$ & $84(22.8)$ & $13(17.1)$ & & & \\
\hline Hemochromatosis & $58(12.4)$ & $30(8.2)$ & $2(2.6)$ & & & \\
\hline Other liver diseases & $58(12.4)$ & $85(23.1)$ & $25(32.9)$ & & & \\
\hline Liver diseases reported as cured & $54(11.6)$ & $15(4.1)$ & $2(2.6)$ & & & \\
\hline Liver transplants & & & & $186(100)$ & & \\
\hline
\end{tabular}

For legend of disease stages, see table I.

based standard errors in SPSS were similar as robust standard errors in SAS.

We evaluated the known groups validity of the LDSI symptom severity items across disease stages and the transplanted group by means of a proportional odds model for ordinal outcome with the PLUM procedure in SPSS 10.0. In every LDSI symptom severity item, the mean probability to score one of the five response categories (1 = 'no symptom' to 5 = 'severe symptom') was estimated per disease stage group or transplant group. We used the same model to evaluate the known groups validity of the extra NLV items.

We evaluated the known groups validity of the LDSI symptom hindrance items across disease stages and the transplanted group by means of binary logistic regression. We estimated for each subgroup the odds ratio of being hampered by symptoms in daily activities (response category 2 to 5 ), relatively to not being hampered (response category 1) by these symptoms. We selected respondents who actually had the symptom (symptom severity $>1$ ), since we assumed that only those respondents could have symptom hindrance.

Estimated differences, probabilities and odds ratios between subgroups were corrected for gender, age, education level, aetiology, use of liver disease medication, use of psychofarmaca and comorbidity. Determinants were regarded as significant when $\mathrm{p}<0.05$.

\section{Results}

\section{Selection of the population}

Of the 2020 members approached for this survey, 1617 members returned the questionnaires. Of these, 374 respondents were non-patient member, who joined the NLV because of involvement with liver patients in family, circle of acquaintances or work. In total 1243 patients had a (history of) liver disease. According to the regulations of the Ethics Committee, we excluded 21 patients who did not give informed consent. Furthermore, we excluded forty-seven patients younger than 18 years of age. In total 1175 respondents were included in the analysis. When we assumed that the percentage of patient members was equal in non-responders and responders $(77 \%)$, than the total number of patient members in the total NLV population would be 1553 and the actual response $(\mathrm{n}=1243)$ would be around $80 \%$.

\section{Baseline characteristics}

Table 2 shows the baseline characteristics of the study population for non-cirrhotic, cirrhotic and transplanted liver patients and the characteristics of Dutch healthy controls for the SF-36 and the MFI-20. The total population of 1175 respondents, of which $678(57.7 \%)$ women, had a mean age of 48.6 years ( $S D \pm 12.7$, range $18-81)$. In total $76 \%$ of these respondents spent on average 24.5 (SD \pm 16.3) hours per week on a paid and/or voluntary job and spent on average $6.5(S D \pm 6.7)$ hours per week on physical activities like walking, cycling and gardening. 
Table 3: Mean SF-36 scores of Dutch healthy controls, non-cirrhotic, compensated cirrhotic, decompensated cirrhotic and transplanted liver patients.

\begin{tabular}{|c|c|c|c|c|c|}
\hline SF-36 scale & $\begin{array}{l}\text { Dutch controls ( } \mathrm{Cl} 95 \%) \\
n=17 / 5\end{array}$ & $\begin{array}{l}\text { LTX (Cl 95\%) } \\
n=186\end{array}$ & $\begin{array}{l}\mathrm{NC}(\mathrm{Cl} \mathrm{95 \% )} \\
\mathrm{n}=489\end{array}$ & $\begin{array}{l}\text { CC (CI 95\%) } \\
n=39 I\end{array}$ & $\begin{array}{l}\mathrm{DC}(\mathrm{Cl} 95 \%) \\
\mathrm{n}=\mathbf{8 4}\end{array}$ \\
\hline PF & $82(8 I-83)$ & $69(66-72)$ & $70(68-72)$ & $65(63-67)$ & $50(66-72)$ \\
\hline $\mathbf{R P}$ & $75(73-76)$ & $53(47-59)$ & $48(45-52)$ & $44(40-48)$ & $21(13-29)$ \\
\hline BP & $74(73-75)$ & $73(69-76)^{*}$ & $66(64-68)$ & $64(6 I-66)$ & $48(43-53)$ \\
\hline GH & 70 (69-7I) & $56(53-59)$ & $46(44-48)$ & $4 \mid(39-43)$ & $31(26-35)$ \\
\hline VI & $67(66-68)$ & $62(59-65)$ & $51(49-53)$ & $50(48-52)$ & $39(35-43)$ \\
\hline SF & $82(8 I-83)$ & $73(69-76)$ & $65(63-67)$ & $64(6 I-66)$ & $47(42-52)$ \\
\hline RE & 80 (78-82) & $74(68-79)$ & $67(63-70)$ & $63(59-67)$ & $49(42-57)$ \\
\hline MH & $75(74-76)$ & 74 (7I-76)* & $67(65-68)$ & $67(66-69)$ & $61(57-65)$ \\
\hline
\end{tabular}

For legend of disease stages, see table I. Legend coding SF36 scales: PF=physical functioning, RP=Role limitations because of physical problems, $\mathrm{BP}=$ bodily pain, $\mathrm{GH}=$ general health, $\mathrm{VI}=$ vitality, $\mathrm{SF}=$ social functioning, $\mathrm{RE}=$ role limitations because of emotional problems, $\mathrm{MH}=\mathrm{mental}$ health. Means are adjusted for gender, age, education level and marital status *) Not significantly different from the score in the general Dutch population.

Table 4: Mean MFI-20 scores of Dutch healthy controls, non-cirrhotic, compensated cirrhotic, decompensated cirrhotic and transplanted liver patients

\begin{tabular}{|c|c|c|c|c|c|}
\hline MFI-20 scales & $\begin{array}{l}\text { Dutch controls (Cl 95\%) } \\
n=139\end{array}$ & $\begin{array}{l}\operatorname{LTX}(\mathrm{Cl} 95 \%) \\
\mathrm{n}=\mathrm{I} 86\end{array}$ & $\begin{array}{l}\mathrm{NC}(\mathrm{Cl} 95 \%) \\
n=489\end{array}$ & $\begin{array}{l}\mathrm{CC}(\mathrm{Cl} 95 \%) \\
\mathrm{N}=39 \mathrm{I}\end{array}$ & $\begin{array}{l}\mathrm{DC}(\mathrm{Cl} 95 \%) \\
n=84\end{array}$ \\
\hline GF & $9.6(8.7-10.4)$ & $11.0(\mid 0.3-11.8)$ & |3.9 (|3.3-14.2) & $14.6(14.1-15.1)$ & $16.6(16.0-17.7)$ \\
\hline PhF & $8.6(7.7-9.4)$ & $11.0(10.2-11.7)$ & $13.0(12.6-13.4)$ & $13.5(13.0-14.0)$ & $16.2(15.2-17.3)$ \\
\hline RA & $8.5(7.7-9.4)$ & $10.1(9.4-10.8)$ & II.5 (II.I-12.0) & $11.7(11.2-12.2)$ & $14.3(13.2-15.3)$ \\
\hline RM & $7.8(7.2-8.7)$ & $8.6(8.0-9.3)^{*}$ & $10.5(10.1-10.9)$ & $10.5(10.0-10.9)$ & $12.4(11.4-13.4)$ \\
\hline MF & $7.9(7.0-8.7)$ & $10.0(9.3-10.7)$ & II.I (I0.6-11.5) & $11.7(\mid 1.2-12.2)$ & $13.3(\mid 2.3-14.5)$ \\
\hline
\end{tabular}

For legend of disease stages, see table I. Legend coding MFI-20 scales: GF=general fatigue, PhF=physical fatigue, RA=reduction in activity, $\mathrm{RM}=$ reduction in motivation, $\mathrm{MF}=$ mental fatigue. Means are adjusted for gender, age and education level *) Not significantly different from the score in the general Dutch population.

All respondents with a liver transplant were assigned to the liver transplant group $(\mathrm{n}=186,16.2 \%)$. The remaining respondents were mainly non-cirrhotic $(42.5 \%)$ and compensated cirrhotic $(34.0 \%)$. Twenty-five respondents were not classified in one of the three disease stage groups or in the transplant group because of missing values in the classification items.

More than one-fifth of the 1175 respondents had viral hepatitis $(23.4 \%)$. Of the $54(4.7 \%)$ respondents categorised as missing, 23 respondents reported cirrhosis as their liver disease, while 31 gave an unclear or insufficient description of their liver disease.

\section{Comparison of generic HRQoL and fatigue with Dutch healthy controls}

Table 3 and 4 show respectively the generic HRQoL and fatigue of chronic liver patients compared to Dutch healthy controls. The majority of the chronic liver patients reported a significantly impaired generic HRQoL and significantly more fatigue compared to healthy controls. Only transplanted liver patients showed a similar level of mental health, bodily pain and reduction in motivation as healthy controls.

\section{Comparison of generic HRQoL and fatigue between non-} cirrhotic, cirrhotic and transplanted patients

Figure 1 shows the mean differences in SF-36 scale scores between non-cirrhotic, cirrhotic and transplanted liver patients. The generic HRQoL of chronic liver patients worsened with a worsening disease stage. Non-cirrhotic and compensated cirrhotic patients showed few significant HRQoL differences. Patients with decompensated cirrhosis mostly demonstrated a significantly worse generic HRQoL than non-cirrhotic patients. In contrast, transplanted patients scored on seven of the eight SF-36 scales a significantly better HRQoL than non-cirrhotic patients. Fatigue showed the same pattern across the disease stages and the transplanted group (figure 2).

\section{Known groups validity of the LDSI items}

Figure 3a to 3i illustrate the known groups validity of the LDSI symptom severity items. The probability to score higher than 1 on itch, pain in the right upper abdomen, 


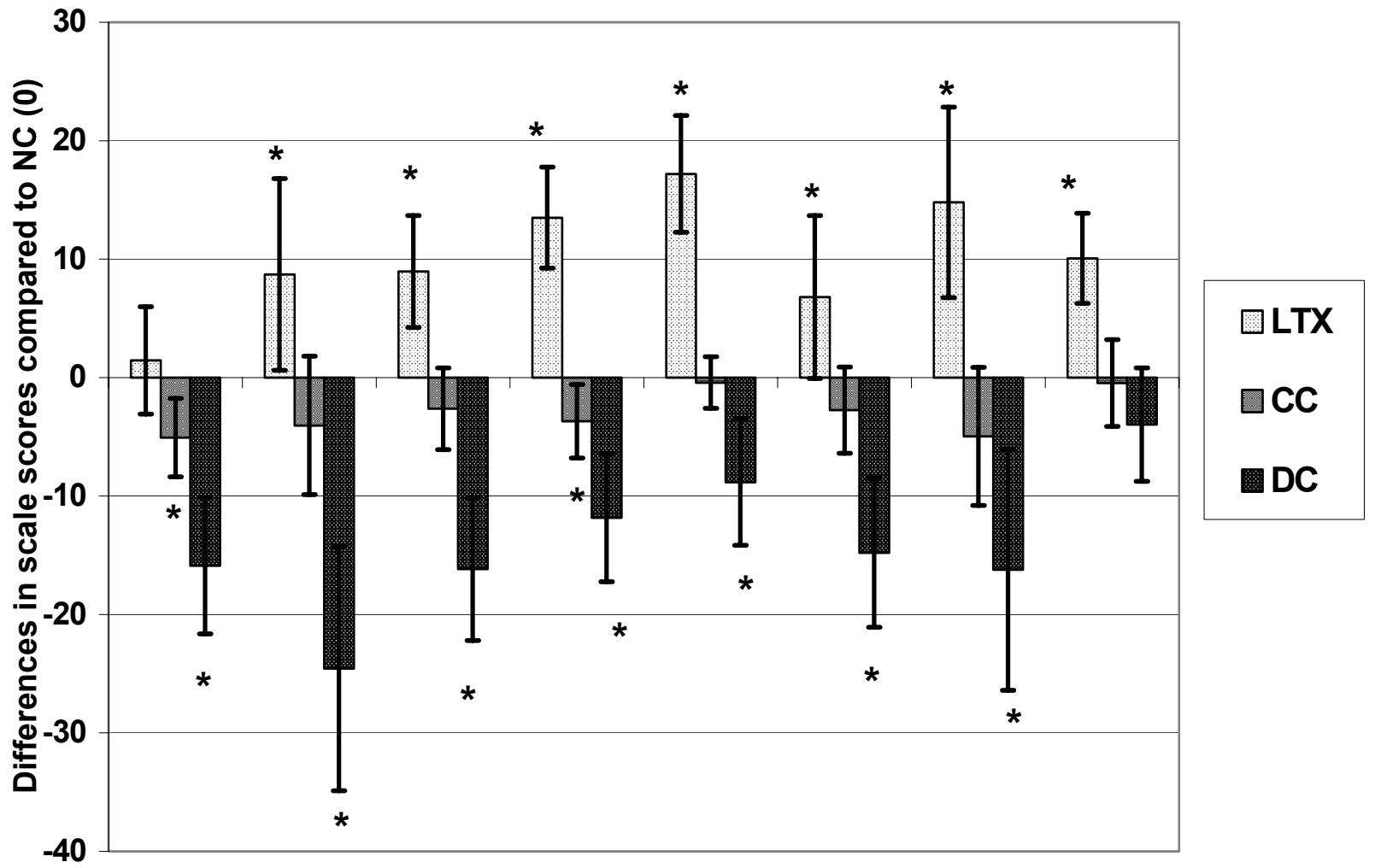

PFRPBPGHVISFREMH

\section{SF-36 scales}

\section{Figure I}

SF-36 scale score differences between non-cirrhotic, compensated cirrhotic, decompensated cirrhotic and transplanted liver patients. For legend of disease stages see table I. For coding of SF36 scales, see legend table 2. Differences are adjusted for gender, age, education level, aetiology, use of liver disease medication, use of psychofarmaca and comorbidity. ${ }^{*}$ ) Scale score of subgroup is significantly different from scale score of NC.

sleepiness, worry about the family situation, decreased appetite, depression, fear and jaundice were highest for liver patients with decompensated cirrhosis. These probabilities were all significantly higher than the probabilities of the non-cirrhotic group ( $p=0.000$ to $p=0.002$ ). Probabilities to score higher than 1 on joint pain were similar for all disease stages. Compensated cirrhotic patients had a significantly higher probability to score higher than 1 on itch $(\mathrm{p}=0.03)$, sleepiness $(\mathrm{p}=0.014)$ and jaundice $(\mathrm{p}=$ $0.008)$ than non-cirrhotic patients. Transplanted liver patients demonstrated significantly lower probabilities to score higher than 1 on itch, joint pain, pain in the right upper abdomen, sleepiness, worry about the family situation, decreased appetite, depression and fear of complications than non-cirrhotic patients $(\mathrm{p}=0.000$ to $\mathrm{p}=0.002)$.

Figure 4 shows the known groups validity of the LDSI symptom hindrance items. Decompensated cirrhotic patients demonstrated for most symptoms significantly higher odds ratios of symptom hindrance than non-cirrhotic patients. Compensated cirrhotic patients showed only significantly higher odds ratios for hindrance of itch during the day and during sleep and hindrance of decreased appetite. Transplanted patients showed a signif- 


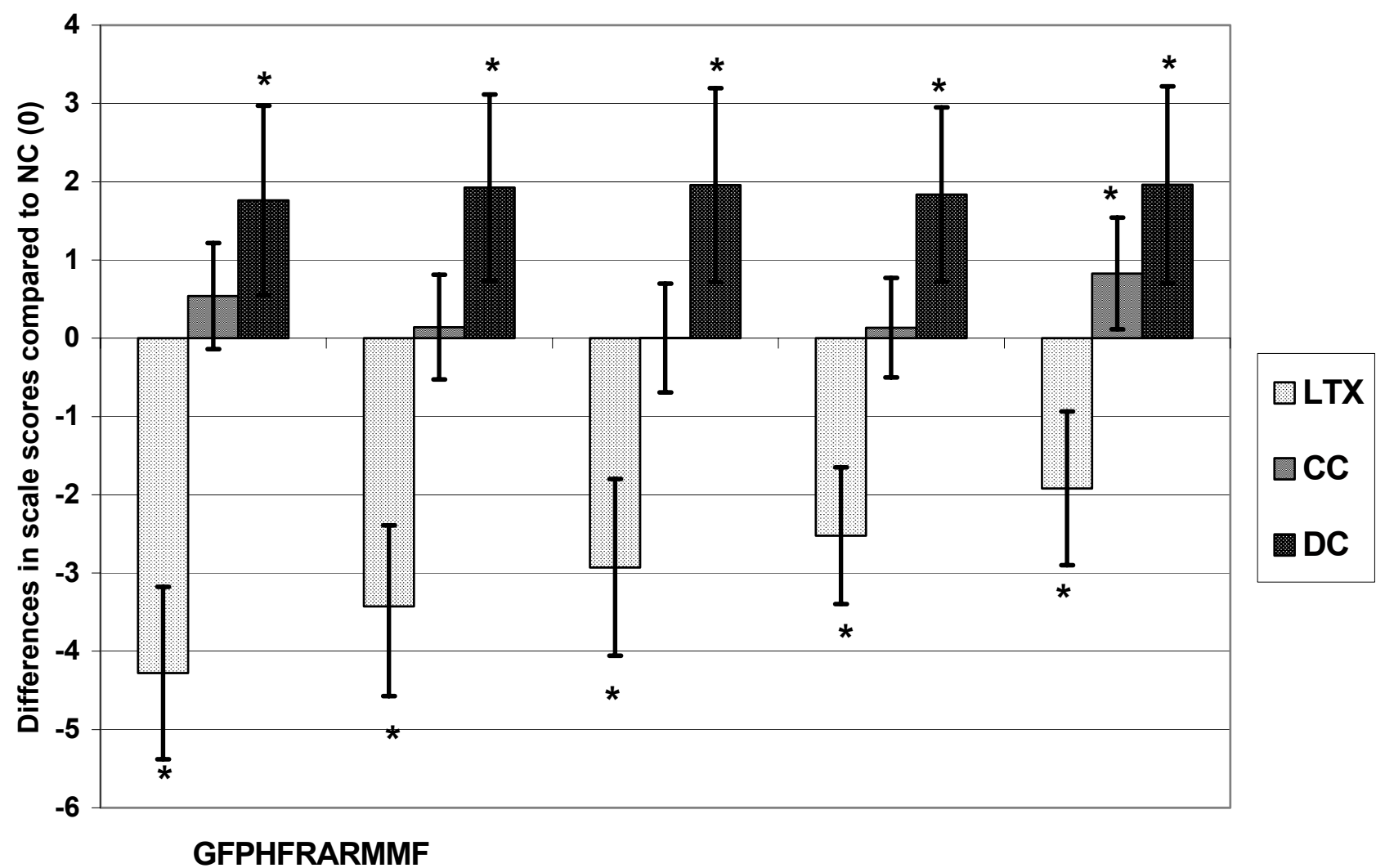

MFI-20 scales

Figure 2

MFI-20 scale score differences between non-cirrhotic, compensated cirrhotic, decompensated cirrhotic and transplanted liver patients. For legend of disease stages, see table I. For coding of MFI-20 scales see legend table 3. Differences are adjusted for gender, age, education level, aetiology, use of liver disease medication, use of psychofarmaca and comorbidity. ${ }^{*}$ ) Scale score of subgroup is significantly different from scale score of NC.

icantly lower odds ratio of hindrance of depression than non-cirrhotic patients.

\section{Known groups validity of extra NLV items}

Finally, we evaluated the known groups validity of the extra NLV items. The analysis showed that decompensated and compensated cirrhotic patients have a significantly higher probability of memory problems than noncirrhotic patients (CC $\mathrm{p}=0.009$, DC $\mathrm{p}=0.00$ ), while transplanted patients show a significantly lower probability $(\mathrm{p}=0.009)$. The probability of a change in personality was, relatively to non-cirrhotic patients, only significantly higher in the compensated and decompensated patient group ( $\mathrm{CC} p=0.011$, DC $\mathrm{p}=0.000)$, although transplanted patients showed a insignificant higher probability compared to non-cirrhotic patients $(\mathrm{p}$ $=0.093)$. Compared to non-cirrhotic patients only decompensated patients showed significantly higher probabilities of financial limitations as a result of the liver disease $(p=0.000)$. Furthermore, the probability of 'involuntary change in use of time' increased significantly with a worsening disease stage $(\mathrm{CC} p=0.019$, $\mathrm{DC} \mathrm{p}=$ $0.000)$. 

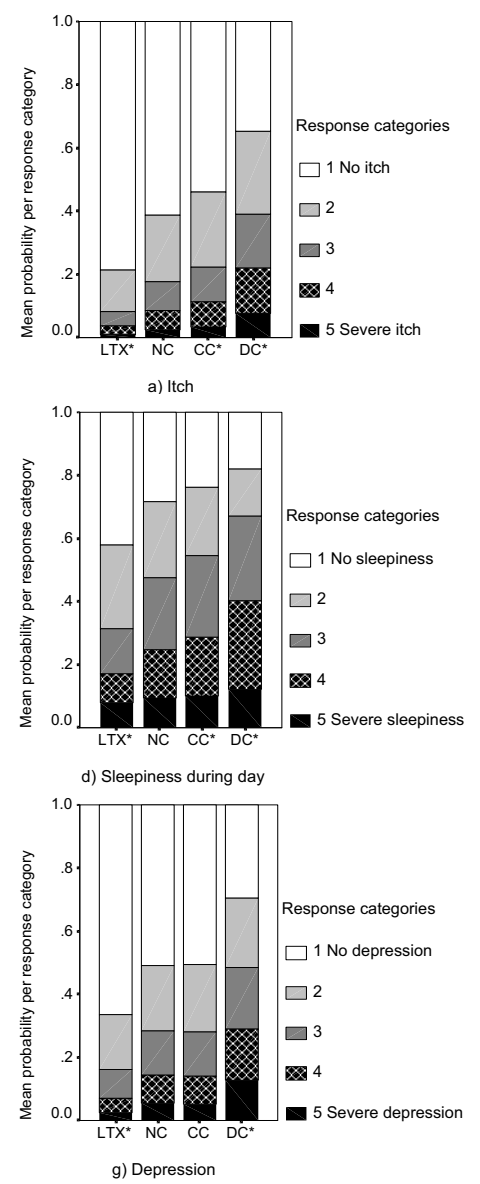
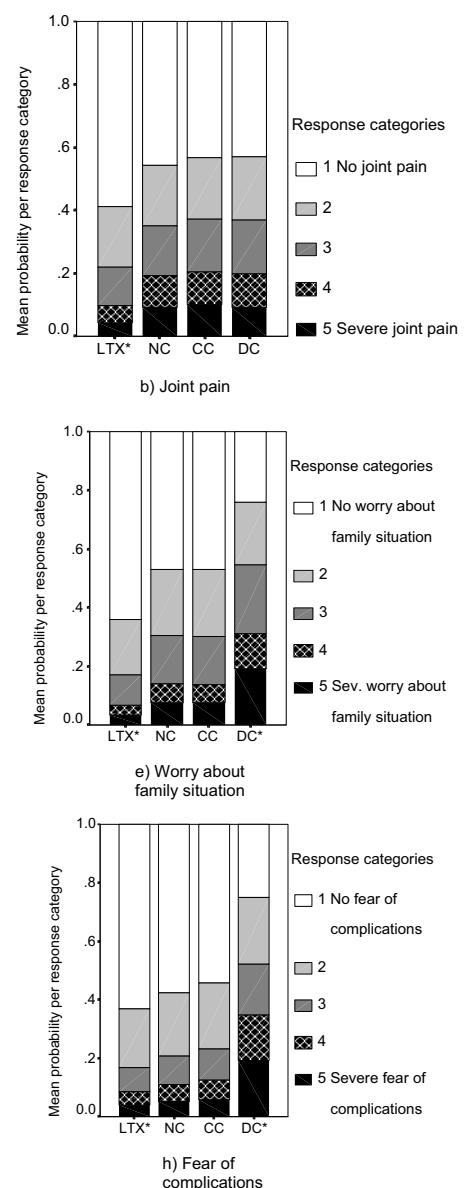
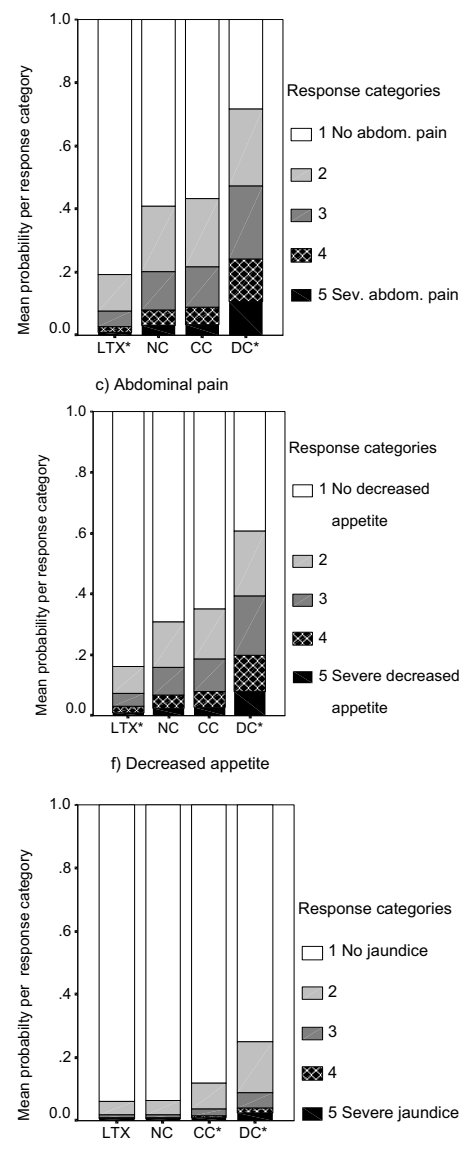

i) Jaundice

\section{Figure 3}

a-i: Probabilities per LDSI symptom severity item per response category, for all liver patients subgroups. For legend of disease stages, see table I. Probabilities are adjusted for gender, age, education level, aetiology, use of liver disease medication, use of psychofarmaca and comorbidity. *)Probabilities of subgroup are significantly different from probabilities of NC.

Transplanted patients showed the lowest probability that the liver disease resulted in 'involuntary change in use of time' $(p=0.000)$. The probabilities of decreased sexual interest were not significantly different between transplanted, compensated cirrhotic and non-cirrhotic patients, but decompensated cirrhotic patients showed a significantly higher probability of decreased sexual interest $(\mathrm{p}=0.000)$. Decompensated as well as transplanted patients showed a significantly higher probability of decreased sexual activity compared to non-cirrhotic patients $(\mathrm{DC} \mathrm{p}=0.016$, LTX $\mathrm{p}=0.001)$.

\section{Discussion}

The aims of this study were: 1) the comparison of the generic HRQoL and fatigue between chronic liver patients and healthy Dutch controls and 2) to give a profound insight in the differences in disease-specific HRQoL, generic HRQoL and fatigue between non-cirrhotic, compensated cirrhotic, decompensated cirrhotic and transplanted liver patients, corrected for various aetiologies.

We have shown that, even after correction for aetiology, generic HRQoL, disease-specific HRQoL and fatigue worsened with a worsening liver disease stage. 


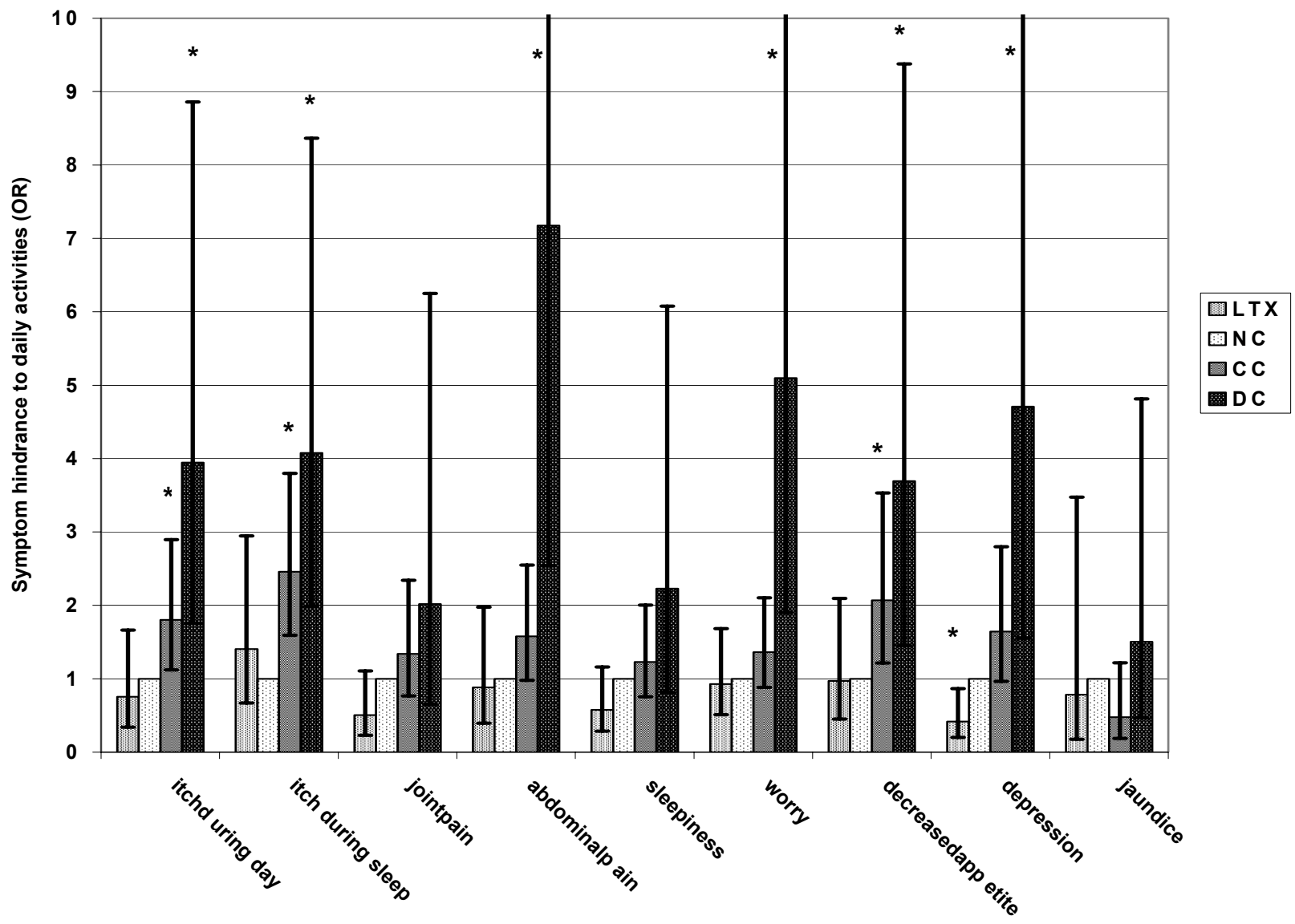

Figure 4

Odds ratios (OR) for symptom hindrance of transplanted and cirrhotic patients compared to non-cirrhotic patients. For legend of disease stages, see table I. *)Odds ratio of subgroup is significantly different from the NC group. Odds ratios are adjusted for sex, age, education level, aetiology, use of liver disease medication, use of psychofarmaca and comorbidity.

However, non-cirrhotic and compensated cirrhotic patients mostly showed insignificant differences with respect to generic and disease-specific HRQoL and fatigue. Decompensated cirrhotic patients revealed a significantly lower generic HRQOL, a higher probability of a worse disease-specific HRQoL and more fatigue than non-cirrhotic patients. Transplanted liver patients demonstrated a better generic HRQoL, a lower probability of severe symptoms and less fatigue than non-cirrhotic and cirrhotic liver patients. However, their probability of symptom hindrance was often not significantly different from the non-cirrhotic group.
The worsening HRQoL across disease stages found in our study, is in line with earlier studies [4-6,14]. Also Unal et al infrequently found significant differences in generic and disease-specific HRQoL and fatigue between non-cirrhotic and compensated cirrhotic patients, although the trend across these two disease stages was reversed (compensated cirrhotic patients showed a better HRQoL than non-cirrhotic) compared to the trend found in our study [9]. Even after we had analysed the Unal data with more advanced statistical methods, corrected for factors like sex, age, education and aetiology, the reversed trend remained. It should however be noted that this study and other earlier studies used different disease stage criteria 
(Child's-Pugh's score and histological data), which hampered the inter-study comparison.

The results of the current study indicate that the LDSI has a moderate to good known groups validity for the three disease stages and the transplanted liver patient group. The symptom severity items easily discriminated the decompensated patients and the transplanted patients from the non-cirrhotic patients. However, difficulties occurred regarding the discrimination between compensated cirrhotic and non-cirrhotic patients. The same problem emerged in the discrimination between compensated and non-cirrhotic patients by the symptom hindrance items.

It is unclear if these difficulties should be attributed to a lack of sensitivity of the LDSI or to the natural characteristics of the compensated cirrhotic disease stage. After all, compensated cirrhotic patients may be asymptomatic for years or decades: Ascites and neurological abnormalities are often absent and in general these patients have a good nutritional state. This may explain the similar HRQoL in non-cirrhotic and compensated cirrhotic patients $[6,15]$. One study already demonstrated the absence of a significant difference in HRQoL between these two groups in a mixed population of chronic liver patients [14]. But a significant difference between the disease-specific HRQoL of non-cirrhotic and compensated cirrhotic was reported as well, although this study only included cholestatic liver patients [5]. Nevertheless, the LDSI items more frequently distinguished between NC and CC patients than the various SF-36 or MFI-20 scales, which illustrated the disease-specific character of the LDSI.

Until now, no other study directly compared the generic and disease-specific HRQoL between transplanted liver patients and non-cirrhotic and cirrhotic liver patients. Earlier studies repeatedly demonstrated that post-transplanted liver patients have a much better HRQoL than pre-transplanted liver patients $[16,17]$. However, our study specifically revealed that transplanted patients also have a better generic HRQoL and less fatigue than non-cirrhotic and cirrhotic liver patients. Nevertheless, the HRQoL of transplanted patients was often significantly impaired, compared to the HRQoL level of the general population.

These results are in line with earlier research, which revealed that transplanted liver patients do have some physical problems, which indeed are experienced as limitations in daily life. Although these limitations barely seem to affect their overall HRQoL as transplanted patients have minimum of concern about physical problems, the presence of limitations may explain the impaired HRQoL of transplanted patients [18-22]. The mental health of transplanted patients was comparable with the mental health of the healthy controls, which confirms earlier literature stating that the tension, depression and anger prevalence rates in transplanted patients were not notably different from the rates in the general population $[23,18]$.

The high HRQoL of transplanted liver patients compared to non-transplanted cirrhotic and non-cirrhotic patients may be explained by the difference in acquired social support. Social support is of utmost importance as a resource of coping with chronic illness and may be beneficial for health outcome regardless of age $[24,25]$. For transplant recipients the psychological support in the transplantation and rehabilitation period provided by medical staff and family, is considered as one of the essentials of the transplant program, as social support influences the post transplantation survival and HRQoL $[18,26]$. However, for other chronic liver patients the enhancement of social support may be less considered as essential part of treatment. Nevertheless, it could positively influence the HRQoL by addressing negative feelings like low selfesteem or hopelessness resulting from the irreversibility of the pathological process and related disability. This potential hiatus in chronic liver disease management could be bridged by Social Network Mapping, which establishes a dialogue regarding individuals' needs and possible sources of support [27].

Despite of the fact that this study included a large population of chronic liver patients, this study design also had certain limitations. Since $90 \%$ of our respondents originated from The Netherlands, our study population could be regarded as a selected population. In another quality of life study conducted at our outpatient clinic, nearly a quarter of the participants were not originally Dutch. Due to the absence of other ethnic groups in our population, extrapolation of our results to outpatient populations should be done with caution.

Additionally, it is unclear which liver patients are attracted by the patient association and how membership influences their HRQoL. Over representation of liver patients with a low HRQoL, seeking contact with other liver patients may have led to an underestimation of HRQoL, while other members' social support may have influenced the measured HRQoL in our population positively. Furthermore, we lacked information about non-responders due to the design of the study. Therefore, responders may have been a selection of relatively healthy patients who felt well enough to complete the questionnaire, which may have led to an overestimation of HRQoL.

A last possible limitation of this study is that we had to depend on the respondents' knowledge with respect to 
data about clinical symptoms and aetiologies. However, our pilot study at the outpatient clinic demonstrated that liver patients are very much aware of the clinical symptoms they have or have had and what type of liver disease they suffer from. As we have no reason to expect that members of a liver patient association are less informed, we are confident that this population based study provided a reliable insight in the HRQOL of chronic liver patients in Western countries.

\section{Conclusions}

We conclude that even after correction for aetiology, the generic and disease-specific HRQoL and fatigue of chronic liver patients depends on the patient's disease stage or transplant history. Although the HRQoL worsened with a worsening disease stage, non-cirrhotic and compensated cirrhotic patients showed barely significant differences in generic and disease-specific HRQoL or fatigue. Decompensated cirrhotic patients showed a significantly worse HRQoL compared to non-cirrhotic patients. The HRQoL of transplanted patients exceeded the HRQoL of all other chronic liver patients, although it was still impaired compared to the HRQoL of healthy controls. Thus, chronic liver patients cannot be considered as one group for whom disease related problems have equal impact on their daily functioning. For a good medical treatment and an honest approach of chronic liver patients it is therefore important that the disease stage or the transplant history are taken into account. Enhancing social support given by medical staff or family as part of chronic liver disease management may partly close the HRQoL-gap between chronic liver patients and transplanted liver patients.

\section{Competing interests}

None declared.

\section{Abbreviations}

HRQoL: Health Related Quality of Life

NLV: Nederlandse Leverpatiënten Vereniging (Dutch liver patient association)

\section{SF-36: Short Form-36}

LDSI: Liver Disease Symptom Index

MFI-20: Multidimensional Fatigue Index-20

NC: Non-Cirrhosis

CC: Compensated Cirrhosis

DC: Decompensated Cirrhosis

LTX: Liver transplant

\section{SD: Standard Deviation}

CI 95\%: 95\% Confidence interval

OR: Odds ratio

\section{Authors' contributions}

SP participated in the design, coordinated the study, performed the statistical analysis and wrote the manuscript.

BH participated in the design of the study and consulted on statistical analysis.

JB consulted on the development and validation of the questionnaires.

TS consulted on the design of the study and statistical analysis.

JP consulted on the design of the study and analysis of questionnaires.

RM established the funding of the study, consulted on design and interpretation of results.

SW consulted on design of the study and interpretation of results.

All authors read and approved the final manuscript.

\section{Acknowledgements}

First, we thank the Dutch Digestive Diseases Foundation (Maag Lever Darm Stichting), for the funding of our study (grant number WS 98-39) and the Dutch liver patient association (Nederlandse Leverpatiënten Vereniging) for their organisational support and their input in the development of various items. Above all, we thank all NLV members for their enthusiastic participation in our study. Without their help this study would not have been possible. Finally, we thank E. Smets and M. Müller for providing respectively the MFI-20 and the SF-36 Dutch norm data.

\section{References}

I. Frequencies of death per primary cause of death and chronic diseases in 2000 [http://statline.cbs.nl/StatWeb]

2. Starzl TE, Koep LJ, Schroter GP, Hood J, Halgrimson CG, Porter KA, Weil $R$ 3rd: The quality of life after liver transplantation. Transplant Proc 1979, I I:252-256.

3. Foster GR, Goldin RD, Thomas HC: Chronic hepatitis C virus infection causes a significant reduction in quality of life in the absence of cirrhosis. Hepatology 1998, 27:209-212.

4. Younossi ZM, Boparai N, Price LL, Kiwi ML, McCormick M, Guyatt G: Health-related quality of life in chronic liver disease: the impact of type and severity of disease. Am J Gastroenterol 200I, 96:2199-2205.

5. Younossi ZM, Kiwi ML, Boparai N, Price LL, Guyatt G: Cholestatic liver diseases and health-related quality of life. $\mathrm{Am} J$ Gastroenterol 2000, 95:497-502.

6. Marchesini G, Bianchi G, Amodio P, Salerno F, Merli M, Panella C, Loguercio C, Apolone G, Niero M, Abbiati R: Factors associated with poor health-related quality of life of patients with cirrhosis. Gastroenterology 200 I, I 20: 170-। 78.

7. Bayliss MS: Methods in outcomes research in hepatology: definitions and domains of quality of life. Hepatology 1999, 29:3S-6S. 
8. Fayers PM, Machin D: Quality of life, assesment, analysis and interpretation Chichester: John Wiley \& Sons, LTD; 2000.

9. Unal G, de Boer JB, Borsboom G], Brouwer JT, Essink-Bot M, de Man RA: A psychometric comparison of health-related quality of life measures in chronic liver disease. J Clin Epidemiol 2001, 54:587-596.

10. Aaronson NK, Muller M, Cohen PD, Essink-Bot ML, Fekkes M, Sanderman R, Sprangers MA, te Velde A, Verrips E: Translation, validation, and norming of the Dutch language version of the SF36 Health Survey in community and chronic disease populations. J Clin Epidemiol 1998, 5 I:1055-1068.

II. Smets EM, Visser MR, Willems-Groot AF, Garssen B, Schuster-Uitterhoeve $A L$, de Haes JC: Fatigue and radiotherapy: (B) experience in patients 9 months following treatment. $\mathrm{Br} J$ Cancer |998, 78:907-9|2.

12. Ware JE Jr, Snow KK, Kosinski M, Gandek B: SF-36 health survey manual and interpretation guide. New England Medical Center, The health institute, Boston 1994

13. Smets EMA, Garssen B, Bonke B: Het meten van vermoeidheid met de multidimensionele vermoeidheidsindex (MVI-20), een handleiding. University of Amsterdam, department of medical psychology 1995.

14. Younossi ZM, Boparai MS, McCormick RN, Price LL, Guyatt G: Assessment of utilities and health-related quality of life in patients with chronic liver disease. Am J Gastroenterol 200I, 96:579-583.

I5. Mclntyre N, Benhamou JP, Bircher J, Rizzetto M, Rodes J: Oxford Textbook of Clinical Hepatology Oxford University Press; I99I.

16. Bravata DM, Olkin I, Barnato AE, Keeffe EB, Owens DK: Healthrelated quality of life after liver transplantation: a meta-analysis. Liver Transpl Surg 1999, 5:3 I8-331.

17. Goff JS, Glazner J, Bilir BM: Measuring outcome after liver transplantation: a critical review. Liver Transpl Surg 1998, 4: I89-196.

18. Kober B, Kuchler T, Broelsch C, Kremer B, Henne-Bruns D: A psychological support concept and quality of life research in a liver transplantation program: an interdisciplinary multicenter study. Psychother Psychosom 1990, 54: | |7-|3|.

19. Robertson G: Individuals' perception of their quality of life following a liver transplant: an exploratory study. J Adv Nurs 1999, 30:497-505.

20. Foley TC, Davis CP, Conway PA: Liver transplant recipients self-report of symptom frequency, symptom distress, quality of life. Transplant Proc 1989, 2 I:24I7-2418.

21. Bonsel GJ, Habbema JD, Bot ML, van 't Veer F, de Charro FT, van der Maas PJ: [Technology assessment of liver transplantation; a study of the liver transplantation program in Groningen 1977-1987]. Ned Tijdschr Geneeskd 1989, I33:|406-14I4.

22. Tarter RE, Switala J, Arria A, Plail J, Van Thiel D: Quality of life before and after orthotopic hepatic transplantation. Arch Intern Med 1991, I5 I:1521-1526.

23. Tarter RE, Erb S, Biller PA, Switala J, Van Thiel DH: The quality of life following liver transplantation: a preliminary report. Gastroenterol Clin North Am 1988, 17:207-217.

24. Siegrist J, Junge A: Measuring the social dimension of subjective health in chronic illness. Psychother Psychosom 1990, 54:90-98.

25. Sherbourne CD, Meredith LS, Rogers W, Ware JE Jr: Social support and stressful life events: age differences in their effects on health-related quality of life among the chronically ill. Qual Life Res 1992, I:235-246.

26. Forsberg A, Backman L, Moller A: Experiencing liver transplantation: a phenomenological approach. J Adv Nurs 2000, 32:327-334.

27. Lewis K, Winsett RP, Cetingok M, Martin J, Hathaway K: Social network mapping with transplant recipients. Prog Transplant 2000, 1 0:262-266

\section{Pre-publication history}

The pre-publication history for this paper can be accessed here:

$$
\text { http://www.biomedcentral.com/1471-230X/3/33/pre }
$$
pub

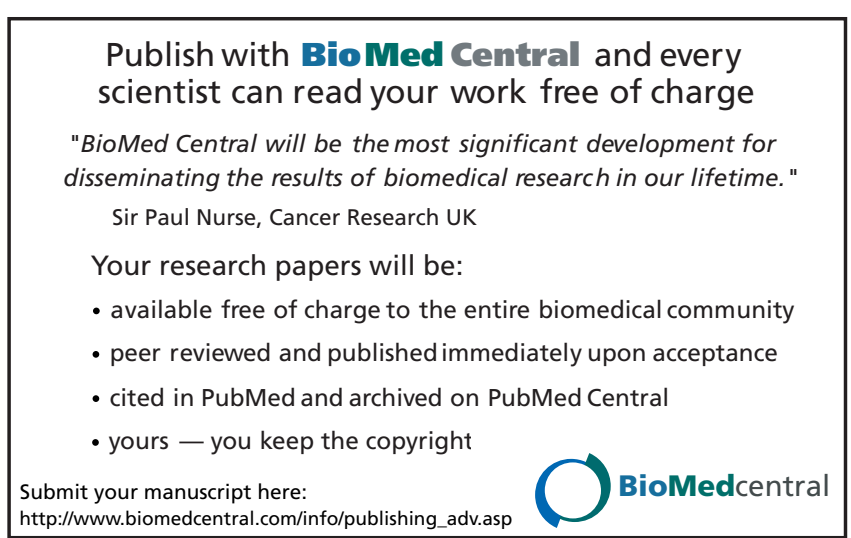

